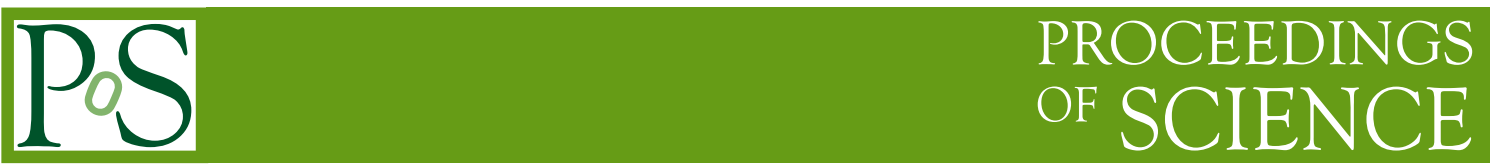

\title{
Lowest energy configurations for Near-BPS Skyrme Models
}

\section{Luc Marleau*t and Nicolas Giasson}

Département de physique, de génie physique et d'optique, Université Laval, Canada

E-mail: lmarleau@phy.ulaval.ca, nicolas.giasson.1@ulaval.ca

\begin{abstract}
The original Skyrme model first proposed over half a century ago, is now understood as a lowenergy effective theory of QCD where baryons (and nuclei) emerge as topological solitons. It provides a relatively good picture of the nucleons, but it overestimates their binding energies in nuclei by at least an order of magnitude. The present work is based on a recent extension of the original Skyrme model, the so-called near-BPS Skyrme Model. As one departs from the pure BPS limit of the model, the solutions nearly saturate the Bogomol'nyi bound which means that the model can be fine-tuned to obtain small binding energies. However, there remains an open question: what solution configuration minimizes the energy? This is of interest since it has some bearing on the nuclei rotational and Coulomb energies. The lack of accuracy of full 3D numerical calculations does not allow for the unambiguous determination of the lowest energy configuration. Here, instead, we compare the two most prominent configurations, the axial and rational map ansatz, for a class of hybrid model that goes from the original to the pure BPS Skyrme model. Our results suggest that the axial solution is the lowest energy configurations for the set of parameters for which the binding energy per nucleon $B / n$ agrees best with the experimental data thereby supporting even further the idea that nuclei could be near-BPS Skyrmions.
\end{abstract}

The European Physical Society Conference on High Energy Physics

22-29 July 2015

Vienna, Austria

${ }^{*}$ Speaker.

${ }^{\dagger}$ Work supported by the NSERC (Canada). 


\section{Introduction}

Despite the successes of the Standard Model, there remains unanswered questions among which one of the most important is the low-energy limit of QCD and its description of the nuclei. The Skyrme Model (1) propose to describe this limit in the form of an effective meson (pion) field theory. The proposal is backed by results on the $1 / N_{c}$ expansion of QCD and more recently by those on holographic QCD (2).The model is usually constructed out of meson fields alone but it can describe baryons and nuclei as well: they arise as topological solitons (Skyrmions) whose winding number corresponds to the baryon number $n$. In fact, the Skyrme Model is rather successful as qualitative description of pion and baryon physics and even at providing quantitative predictions which are within at least $30 \%$ of accuracy (often to a few \%) when compared to experimental data. Unfortunatly, in its most naive version, it fails to give an appropriate account of multibaryon physics or nuclei, especially for binding energies, which are too large (e.g. deuteron $\simeq 40 \times$ observed value). In addition, there are a number of technical difficulties linked to the nature of the model such as its non pertubative regime and non linearity. As a result, finding the lowest energy configurations is numerically challenging so that only some low $n$ solutions are known (e.g. toroidal, tetrahedral, cubic configurations for $n=2,3,4$ Skyrmions, respectively). Yet, the analysis of various potential (mass) terms, rotational deformations, higher order terms in derivatives (3) and additional mesons (e.g. $\omega, \rho, \ldots$ ) lead, in general, to similar configurations and binding energies.

However, it was realized recently $(4 ; 5)$ that if the model was to be constructed along the lines of a BPS model it would have zero or small binding energies since the mass of a nucleus is almost proportional to that of the nucleon, $M_{\text {nuclei }} \approx n \cdot M_{\text {nucleon }}$, and we know that BPS-solitons follow this exact pattern. The present work is based on a recent extension of the original Skyrme Model, the so-called near-BPS Skyrme Model (5; 6), which have by construction small binding energies. However, there remains an open question: what solution configuration minimizes the energy? A complete analysis of this class of models and parameters on which it depends is numericallly prohibitive so here instead, we aim for a simpler prospective analysis and confine our analysis to two simple ansatzes. The original Skyrme Model leads to configurations that are close to that of the rational map ansatz (RM) whereas some solutions of the pure BPS Skyrme Model (4) have axial symmetry (Ax). Our goal is to compare these two configurations (axial and rational map ansatz) for a class of hybrid model that goes from the original Skyrme Model to the pure BPS Skyrme Model (4) and in doing so, determine in which domain of model parameters each configuration remains appropriate and is most relevant.

The next figure present the baryon density configurations for the axially symmetric (Ax), rational map (RM) ansatzes considered in this work as well as Crystal Like structure ( CS) that emerges for some extensions of the Skyrme Model.

Ax

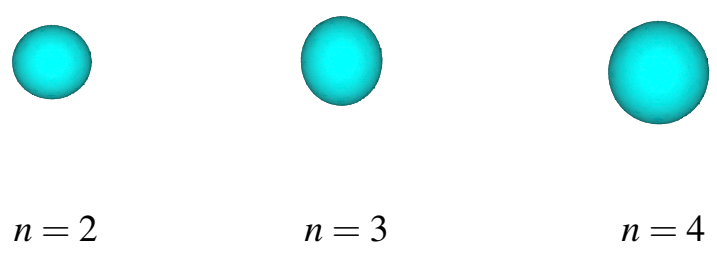


$\mathrm{RM}$

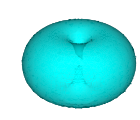

CS

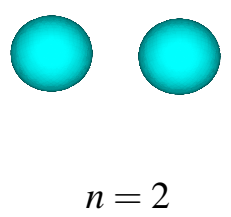

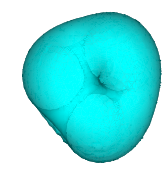

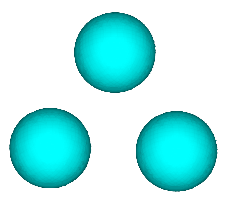

$n=3$
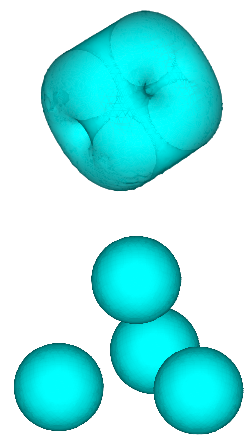

$n=4$

\section{Near-BPS Skyrme Models}

We consider a Skyrme-like model in a regime where the solutions are near-BPS solitons so that $M_{\text {nuclei }} \approx n \cdot M$. It is described by the Lagrangian density

$$
\mathscr{L}_{\mathrm{NBPS}}=\underbrace{\mathscr{L}_{0}+\mathscr{L}_{6}}_{\text {BPS-solitons }}+\underbrace{\mathscr{L}_{2}+\mathscr{L}_{4}}_{\text {Skyrme }} \text {. }
$$

The pion fields are introduced through $\phi_{i}$ in $U=\phi_{0}+i \tau_{i} \phi_{i}$ where $U \in S U(2)$ and for simplicity we write $L_{\mu}=U^{\dagger} \partial_{\mu} U$ and $f_{\mu \nu}=\left[L_{\mu}, L_{v}\right] . \mathscr{L}_{\mathrm{NBPS}}$ contains the standard nonlinear- $\sigma$ model and Skyrme terms, written respectively as,

$$
\mathscr{L}_{2}=-\alpha \operatorname{Tr}\left[L_{\mu} L^{\mu}\right], \mathscr{L}_{4}=\beta \operatorname{Tr}\left(f_{\mu v}^{2}\right),
$$

and what we will assume to be the dominant terms in the near-BPS approach, the potential term $\mathscr{L}_{0}=-\mu^{2} V(U)$ and the sextic term

$$
\mathscr{L}_{6}=-\left(3 \lambda^{2} / 512\right) \operatorname{Tr}\left(f_{\mu v} f^{\nu \lambda} f_{\lambda}^{\mu}\right) .
$$

In the near-BPS approach, the terms $\mathscr{L}_{0}+\mathscr{L}_{6}$ dominate, treating $\mathscr{L}_{2}$ and $\mathscr{L}_{4}$ as small perturbations. Finite energy solutions require a conserved topological charge $\left(\mathscr{B}^{0}=\right.$ baryon density)

$$
n=\int d^{3} r \mathscr{B}^{0}=-\frac{\varepsilon^{i j k}}{24 \pi^{2}} \int d^{3} r \operatorname{Tr}\left(L_{i} L_{j} L_{k}\right)
$$

When $\alpha=\beta=0$, these solutions are BPS-solitons so their masses are exactly proportional to $n$.

In this work we analyze solutions based on two ansatzes: The first one is the axially symmetric (Ax) ansatz (4) which is a lowest energy solution and a BPS soliton when $\alpha=\beta=0$. It reads

$$
U=\cos (F(r)) \mathbb{I}_{2}+i \hat{\mathbf{n}} \cdot \tau \sin (F(r)),
$$

where $F(r)$ encodes the radial dependence and the unit vector $\hat{\mathbf{n}}$ the angular part of the solution

$$
\hat{\mathbf{n}}=(\sin \theta \cos n \phi, \sin \theta \sin n \phi, \cos \theta) .
$$

Note that for $n>1$, the absolute static energy minimizer for $\alpha, \beta \neq 0$ is conjectured to be "restricted harmonic map" (7). 
The second ansatz of interest is the rational map (RM) ansatz. It is near the static energy minimizer of the Skyrme Model $\left(\mathscr{L}_{2}+\mathscr{L}_{4}\right)$ or when $\mu, \lambda$ are small. The rational map ansatz stands for

$$
\hat{\mathbf{n}}=\frac{1}{1+R \bar{R}}(R+\bar{R},-i(R-\bar{R}), 1-R \bar{R}) .
$$

where the angular dependence is encoded in $R(z)=\frac{p(z)}{q(z)}$ with $z=\tan \frac{\theta}{2} e^{i \phi}$ and where $p(z)$ and $q(z)$ are polynomials of degree $n$. The static energy minimizer of the Skyrme Model correspond to $R(z)$

$$
R(z)=z, z^{2}, \frac{z^{3}-\sqrt{3} i z}{\sqrt{3} i z^{2}-1}, \frac{z^{4}+2 \sqrt{3} i z^{2}+1}{z^{4}-2 \sqrt{3} i z^{2}+1}, \ldots \text { for } n=1,2,3,4, \ldots
$$

The Ax and RM ansatzes allow to integrate over the angular dependence and write the total static energy using $E_{\text {stat }}=E_{0}+E_{2}+E_{4}+E_{6}$ with

$$
\begin{gathered}
E_{0}=4 \pi \mu^{2} \int V(F) r^{2} d r \quad E_{2}=8 \pi \alpha \int\left(F^{\prime 2} r^{2}+f_{1} \sin ^{2}(F)\right) d r \\
E_{4}=64 \pi \beta \int\left(f_{1} \sin ^{2}(F) F^{\prime 2}+f_{2} \frac{\sin ^{4}(F)}{r^{2}}\right) d r \quad E_{6}=\frac{9 \pi \lambda^{2} f_{2}}{4} \int \frac{\sin ^{4}(F) F^{\prime 2}}{r^{2}} d r,
\end{gathered}
$$

Here $f_{1}=n^{2}+1$ and $2 n$ and $f_{2}=n^{2}$ and $\mathscr{I}$ for Ax and RM respectively where the angular integral $\mathscr{I}$ is defined as

$$
\mathscr{I} \equiv \frac{1}{4 \pi} \int\left(\frac{(1+z \bar{z})^{2}}{(1+R \bar{R})^{2}} \frac{d R}{d z} \frac{d \bar{R}}{d \bar{z}}\right)^{2} \frac{2 i}{(1+z \bar{z})^{2}} d z d \bar{z}
$$

Other energetic contributions, such as rotational energy, isospin energy and Coulomb energy can also be computed in a similar fashion. However, since they do not account for a significant portion of the total mass of a nucleus, and that we wish to highlight the differences between the minimal energy configurations of the axially symmetric and rational map solutions for the deuterium $(n=2)$ and helium-4 $(n=4)$ nuclei, these aforementioned energetic contributions will not be include here.

There remains one important ingredient that is still missing for an explicit computation of the static energy $E_{\text {stat }}$, the potential $V(F)$. Here, we are interested in a model that can reproduce the constant baryon density in nuclei. So we proceed as follows: First, we introduce an appropriate $V(F)$ in order to generate constant baryon density such as in Ref. (6). Then, we write $E_{\text {stat }}$ in terms of a new parameter $v$, in order to adjust the relative strength of the pure BPS and Skyrme Lagrangians sub-models

$$
E_{\text {stat }}(v)=(1-v) E_{0}+v E_{2}+v E_{4}+(1-v) E_{6},
$$

Finally, we use numerical analysis to minimize the static energy for the entire $v$ spectrum with a simulated annealing algorithm that finds the minimizing profile $F(r)$ directly. Note that the limit $v \rightarrow 0$ corresponds to the pure BPS model, which can be solved analytically in both cases leading to $F(r)=2 \arcsin \left(\exp \left[-a^{2} r^{2}-\frac{7}{5} a^{4} r^{4}\right]\right)$ where $a^{3}=(\mu / 18 n \lambda)$ and $(\mu / 18 \sqrt{\mathscr{I}} \lambda)$ for Ax and RM respectively. 


\section{Results and Conclusion}

We present the results in the terms of the quantity $\mathrm{R}_{n}^{v}$, the ratio of the static energy of a nuclei with atomic number $n$ over that of the nucleon as a function of the parameter $v$,

$$
\mathrm{R}_{n}^{v}=\frac{E_{\text {stat }}(v, n)}{E_{\text {stat }}(v, n=1)}
$$

For example, in the limit $v \rightarrow 0$, the ratio is given by $\mathrm{R}_{n}^{0}=n$ and $\sqrt{\mathscr{I}}$ for Ax and RM respectively. The results for $n=2$ for values of $0 \leq v \leq 1$ are shown in figure 1 .

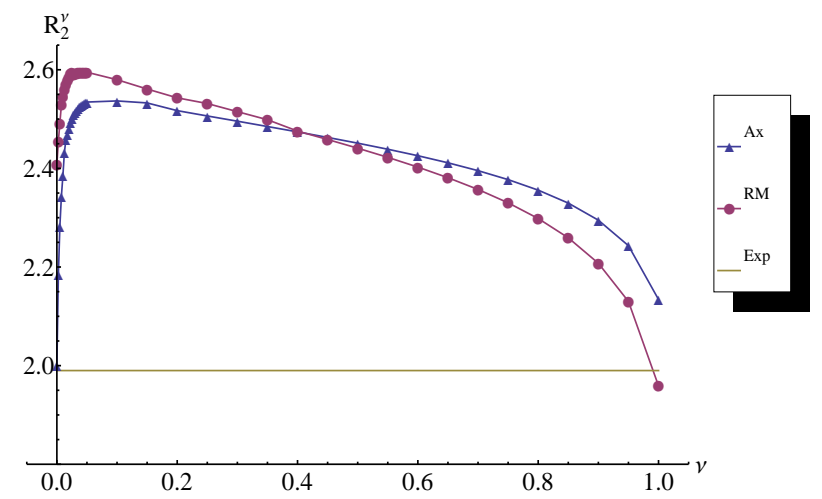

Figure 1: Ratios $\mathrm{R}_{n}^{v}$ for deuterium ( $n=2$ ) as a function of $v$ for the axially symmetric (Ax) and rational map (RM) ansatzes.

A number of conclusions can be drawn from our results: (a) We find that there is a bound state at the classical level for $v=1$ (dot below the $\mathrm{R}_{2}^{v}=2$ limit) with the rational map ansatz but this is not new information since this case was well known for the Skyrme Model. (b) A bound state is also possible if we have $\alpha \gg \beta, \beta \sim 10^{-8}$ (very small $v$ values) while adding other energetic contributions. (c) Thus, further analyses of these results demonstrate that a minimization of the form $E_{\text {stat }}=(1-v) E_{0}+v E_{2}+\nu E_{4}+(1-v) E_{6}$ also lead to bound states as can be shown from figure.2. Similar relations and conclusions are obtained for the case of helium-4 $(n=4)$ but they are not shown here due to space constraints.

Our analysis also suggests that: (a) Removing the $\mathscr{L}_{4}$ term in the near-BPS Lagrangian does not destabilize the soliton solutions since the $\mathscr{L}_{6}$ term can account for this specific task. (b) Bound states (stable states) can be observed in both finite regions near $v=0$ and $v=1$. Moreover, in the region dominated by the BPS model $(0 \leq v \leq 0.5)$, these bound states are best described by the axially symmetric ansatz (lower ratios). Provided that we set $\beta=0$ in $E_{\text {stat }}$, the perturbative approach can be used in conjunction with the axially symmetric ansatz to describe nuclei (with the advantages of analytic solutions). Given our choice of potential and parameters, we find a very small value for the pion decay constant $F_{\pi}=0.09 \mathrm{MeV}\left(F_{\pi}^{\text {expt }}=186 \mathrm{MeV}\right)$ and no pion mass $m_{\pi}=0\left(m_{\pi}^{\text {expt }}=138 \mathrm{MeV}\right)$ so the link to soft-pion physics is yet unclear.

The goal of this work was to find the best parametric representation of $U$ that leads to minimal energetic configurations of the near BPS-Skyrme Model. This remains an important question since the real structure of nuclei is still unknown and, as we have seen, the choices we make directly 


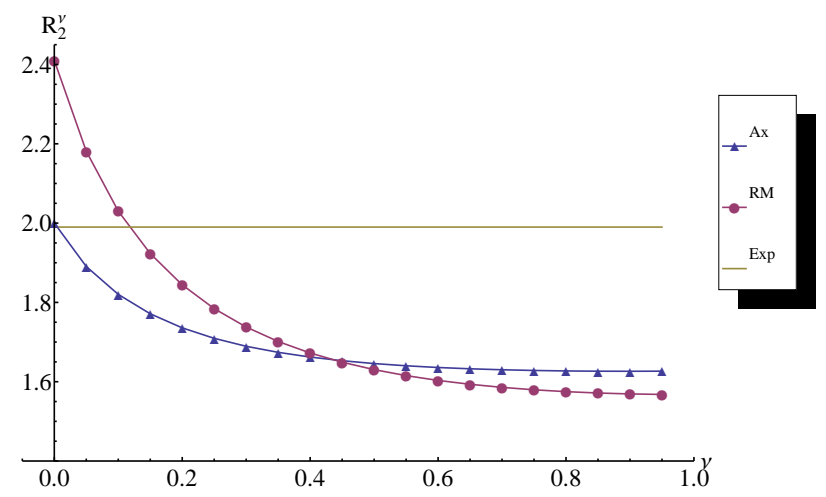

Figure 2: Ratios $\mathrm{R}_{n}^{v}$ for deuterium ( $\left.n=2\right)$ as a function of $v$ for the axially symmetric (Ax) and rational map (RM) ansatzes for the case $\beta=0$ (no $E_{4}$ term). A bound state exists if $\mathrm{R}_{n}^{v}<n$.

affect the resulting energy and geometry of the solutions in this model. A suitable parametrization could indeed lead to an improvement of the predictive power of the model.

The exact solution that minimizes the static energy (in this case the mass of the nuclei) for a given $n$ remains unknown. Our work however indicates that the rational map configuration are not very relevant for the near-BPS model at hand. Of course, several aspects of the model remain to be studied (e.g. magnetic moments, vibrational and rotational excitations....)

\section{References}

[1] T.H.R. Skyrme, A Nonlinear field theory, Proc. R. Soc. A260, 127 (1961)

[2] T. Sakai and S. Sugimoto, Low energy hadron physics in holographic QCD, Prog. Theor. Phys. 113, 843 (2005); P. Sutcliffe, Multi-Skyrmions with Vector Mesons, Phys. Rev. D79, 085014 (2009).

[3] L. Marleau, Modifying the Skyrme model: Pion mass and higher derivatives, Phys. Rev. D43, 885 (1991); B. Dion, L. Marleau and G. Simon, Skyrmions from a Born-Infeld action, Phys. Rev. D53, 1542 (1996); L. Marleau and J. F. Rivard, A generating function for all orders skyrmions, Phys. Rev. D63, 036007 (2001).

[4] C. Adam, J. Sanchez-Guillen and A. Wereszczynski, A Skyrme-type proposal for baryonic matter, Phys. Lett. B691 :105-110, 2010.

[5] E. Bonenfant, L. Marleau, Nuclei as near BPS-Skyrmion, Phys. Rev. D82, 054023 (2010) ;

E. Bonenfant, L. Harbour, L. Marleau, Near-BPS Skyrmions: Non-shell configurations and Coulomb effects, Phys. Rev. D85, 114045 (2012).

[6] M.O. Beaudoin, L. Marleau, Near-BPS Skyrmions: Constant baryon density, Nucl. Phys. B883 (2014) 328-349.

[7] J. M. Speight, Near BPS Skyrmions and Restricted Harmonic Maps, J. Geom. Phys. 92, 30 (2015). 